\title{
Association study of essential tremor genetic loci in Parkinson's disease
}

\author{
Jay P. Ross ${ }^{\mathrm{a}, \mathrm{b}}$, Sadaf Mohtashami ${ }^{\mathrm{b}, \mathrm{c}}$, Etienne Leveille ${ }^{\mathrm{d}}$, Amelie M. Johnson ${ }^{\mathrm{e}}$, Lan Xiong e,f \\ Patrick A. Dion ${ }^{\mathrm{b}, \mathrm{g}}$, Edward Fon ${ }^{\mathrm{b}, \mathrm{c}}$, Yves Dauvilliers ${ }^{\mathrm{h}}$, Nicolas Dupré ${ }^{\mathrm{i}, \mathrm{j}}$, Guy A. Rouleau ${ }^{\mathrm{a}, \mathrm{b}, \mathrm{g}}$, \\ Ziv Gan-Or ${ }^{\mathrm{a}, \mathrm{b}, \mathrm{g}, *}$
}

${ }^{a}$ Department of Human Genetics, McGill University, Montréal, Quebec, Canada

${ }^{\mathrm{b}}$ Montreal Neurological Institute, McGill University, Montréal, Quebec, Canada

${ }^{\mathrm{c}}$ Department of Experimental Medicine, McGill University, Montréal, Quebec, Canada

${ }^{\mathrm{d}}$ Faculty of Medicine, McGill University, Montréal, Quebec, Canada

e Laboratoire de neurogénétique, Centre de recherche, Institut universitaire en santé mentale de Montréal, Montréal, Quebec, Canada

${ }^{\mathrm{f}}$ Département de psychiatrie, Université de Montréal, Montréal, Quebec, Canada

${ }^{\mathrm{g}}$ Department of Neurology and Neurosurgery, McGill University, Montréal, Quebec, Canada

${ }^{\mathrm{h}}$ National Reference Center for Narcolepsy, Sleep Unit, Department of Neurology, Gui-de-Chauliac Hospital, CHU Montpellier, University of Montpellier, Inserm U1061, Montpellier, France

${ }^{\mathrm{i}}$ Division of Neurosciences, CHU de Québec, Université Laval, Quebec City, Quebec, Canada

${ }^{\mathrm{j}}$ Department of Medicine, Faculty of Medicine, Université Laval, Quebec City, Quebec, Canada

\section{A R T I C L E I N F O}

\section{Article history:}

Received 30 September 2017

Received in revised form 17 December 2017

Accepted 1 January 2018

Available online 6 January 2018

\section{Keywords:}

Parkinson's disease

Essential tremor

GWAS

\begin{abstract}
A B S T R A C T
A recent genome-wide association study identified variants associated with essential tremor (ET). The present study aimed to examine potential genetic overlap between ET and Parkinson's disease (PD). The top 22 variants identified by the ET genome-wide association study and 4 additional variants from previous studies were genotyped in a cohort of French and French-Canadian PD patients $(n=717)$ and controls $(n=595)$. Logistic regression analysis, adjusted for age and sex, was used to test for association between genotype and PD. None of the variants tested in the present study was significantly associated with PD. Our results do not support a role of ET-associated genetic variants in PD.
\end{abstract}

(c) 2018 Elsevier Inc. All rights reserved.

\section{Introduction}

Parkinson's disease (PD) and essential tremor (ET) are common movement disorders that affect a significant proportion of the elderly population. ET generally presents as an action tremor without neurodegeneration, whereas PD presents as a resting tremor, along with other motor and nonmotor symptoms. Interestingly, several studies suggested an overlap between ET and PD risk. The risk to develop PD after an initial diagnosis of ET was 4-fold when compared to a non-ET population (Algarni and Fasano, 2017), and individuals with a PD-diagnosed relative are more likely (depending on age and gender) to develop ET (Rocca et al., 2007). While the 2 diseases

\footnotetext{
* Corresponding author at: Montreal Neurological Institute and Hospital, Department of Neurology \& Neurosurgery, Department of Human Genetics, McGill University, 1033 Pine Avenue West, Ludmer Pavilion, room 327, Montreal, QC H3A 1A1, Canada. Tel.: +1 514398 5845; fax: +1 5143988248 .

E-mail address: ziv.gan-or@mcgill.ca (Z. Gan-Or).
}

are mostly distinct in their etiology and symptoms, there may be potential genetic pleiotropy between the diseases (Rocca et al., 2007). A recent genome-wide association study (GWAS) has suggested associations between several loci and ET (Muller et al., 2016). Because the clinical and genetic link between PD and ET are still not fully understood, we screened variants from the recent ET GWAS (Muller et al., 2016) in a cohort of French and French-Canadian PD patients and unaffected controls. See Supplementary Material for detailed introduction and full list of references.

\section{Methods}

\subsection{Samples}

A case-control series consisting of 717 subjects with PD (average age $65.94 \pm 9.42$ years, 1.79 male-female ratio) and 595 unrelated, unaffected controls ( $51.68 \pm 13.14$ years, 1.11 male-female ratio) was included in this study. Details on recruitment and detailed methods 
can be found in the Supplementary Material. All subjects provided informed consent, and the study was approved by the respective institutional review boards.

\subsection{Variant selection}

The top 22 single-nucleotide polymorphisms (SNPs) most significantly associated with ET identified in a previous GWAS (Muller et al., 2016) were included (Table 1). Four SNPs from previous studies with possible association to ET were also included.

\subsection{Genotyping}

Variants were genotyped on a custom-designed TaqMan OpenArray Genotyping platform using a standard protocol and analyzed using Quantstudio 12K Flex Software v1.2.2 and TaqMan Genotyping Software v1.3.1. Two variants were genotyped using TaqMan Genotyping following standard protocols.

\subsection{Statistical analysis}

Quality control and statistical analysis was performed using PLINK 1.9 and included per-sample call rate $>0.90$ and lack of deviation from Hardy-Weinberg equilibrium. Binary logistic regression was used to test for association, with age and sex as covariates. Association was considered significant below a Bonferroni multiple testing threshold of $p<0.05 / 26$ (0.0019).

\section{Results}

A total of 56 samples (0.036) were excluded from subsequent analysis as they had genotyping call rates $<0.90$. Hence, a total of 679 PD patients and 577 controls were included in the regression model, and the final genotyping call rate across all samples was 0.995. No variants significantly deviated from Hardy-Weinberg equilibrium (all had $p>0.01$ ). Table 1 details the association of the ET SNPs with PD in our cohort. Following multiple testing correction, none of the variants were found to be significantly associated with PD (Table 1). Further supporting lack of association, all the tested SNPs had corrected $p>0.05$ in PDGene (www. pdgene.org).

\section{Discussion}

Our results do not support pleiotropy between ET and PD, based on the top 22 SNPs identified in the ET GWAS (Muller et al., 2016) and 4 other SNPs from previous studies. As the recent GWAS identified loci associated with ET, if a significant overlap between ET and PD exists, there should be some variants that are common to both diseases. As patients who where initially diagnosed as ET have a risk to be later diagnosed as having PD (Lees et al., 2009), variants observed in both diseases may be predictive for this conversion. However, we did not find evidence for this possibility. Furthermore, when examining the PDGene database (www. pdgene.org), which summarizes data from large PD GWAS including 13,708 PD patients and 95,282 controls, all the tested SNPs had corrected $p$ values of $>0.05$, further demonstrating lack of pleiotropy between ET and PD. Interestingly, one of the top ETassociated SNPs is located near the RAB29 gene within the PARK16 locus, which is strongly associated with PD. However, rs823141, possibly associated with ET (odds ratio $=1.17 ; 95 \%$ confidence interval $=1.10-1.25 ; p=1.54 \times 10^{-6}$ in the ET study), is not associated with PD in our cohort and only has nominal association $(p<0.05)$ in the PDGene database. Of note, that the direction of effect is opposite within this locus, as in ET, the minor allele is associated with increased risk, and in PD, it is associated with decreased risk. It is therefore possible that different alleles or genetic risk factors are associated with ET and PD within this locus, and further genetic analysis is needed. A more detailed discussion including full list of references can be found in the Supplementary Material.

Table 1

ET-associated variants in 679 Parkinson's disease patients and 577 controls

\begin{tabular}{|c|c|c|c|c|c|c|c|}
\hline CHR:POS & $\mathrm{dbSNP}$ & NT & Gene & $\operatorname{MAF}(\mathrm{A})$ & $\operatorname{MAF}(\mathrm{U})$ & OR $(95 \% \mathrm{CI})$ & $p$ value \\
\hline $1: 205741426$ & rs823141 & $\mathrm{T} / \mathrm{C}$ & RAB29 & 0.480 & 0.455 & $1.089(0.8956-1.325)$ & 0.3919 \\
\hline $1: 90646872$ & rs6675307 & $\mathrm{G} / \mathrm{A}$ & Intergenic & 0.240 & 0.221 & $1.039(0.8226-1.312)$ & 0.7484 \\
\hline $2: 12186335$ & rs893787 & $\mathrm{T} / \mathrm{C}$ & LOC100506457 & 0.509 & 0.482 & $1.093(0.8981-1.331)$ & 0.3744 \\
\hline $2: 19901053$ & rs34533275 & $\mathrm{T} / \mathrm{C}$ & Intergenic & 0.319 & 0.299 & $1.01(0.8184-1.246)$ & 0.9264 \\
\hline $2: 203750049$ & rs10189499 & $\mathrm{T} / \mathrm{C}$ & WDR12 & 0.360 & 0.332 & $1.078(0.8793-1.322)$ & 0.4691 \\
\hline $2: 220050707$ & rs11680709 & $\mathrm{A} / \mathrm{G}$ & HS1-BP3 & 0.425 & 0.406 & $1.085(0.8903-1.323)$ & 0.4180 \\
\hline $2: 235807629$ & rs6431308 & $\mathrm{A} / \mathrm{C}$ & Intergenic & 0.241 & 0.232 & $1.132(0.8951-1.432)$ & 0.3007 \\
\hline $3: 113890815$ & rs6280 & $\mathrm{T} / \mathrm{C}$ & DRD3 & 0.312 & 0.296 & $1.052(0.8504-1.302)$ & 0.6390 \\
\hline $3: 151563759$ & rs10935878 & $\mathrm{T} / \mathrm{A}$ & AADACL2-AS1 & 0.290 & 0.263 & $1.203(0.9646-1.5)$ & 0.1011 \\
\hline $4: 177242959$ & rs4690686 & $\mathrm{C} / \mathrm{T}$ & SPCS3 & 0.430 & 0.382 & $1.125(0.9227-1.372)$ & 0.2440 \\
\hline $4: 24362541$ & rs17590046 & $\mathrm{T} / \mathrm{C}$ & PPARGC1A & 0.200 & 0.215 & $0.9571(0.7489-1.223)$ & 0.7258 \\
\hline $4: 5128159$ & rs10937625 & $\mathrm{T} / \mathrm{C}$ & STK32B & 0.247 & 0.271 & $0.8843(0.7071-1.106)$ & 0.2813 \\
\hline 4:79421963 & rs1496588 & $\mathrm{T} / \mathrm{C}$ & FRAS1 & 0.483 & 0.486 & $0.9839(0.8115-1.193)$ & 0.8691 \\
\hline 6:33778964 & rs9394169 & $\mathrm{G} / \mathrm{A}$ & Intergenic & 0.474 & 0.462 & $1.097(0.901-1.335)$ & 0.3573 \\
\hline $7: 115554668$ & rs2402000 & $\mathrm{C} / \mathrm{T}$ & Intergenic & 0.247 & 0.234 & $1.091(0.8651-1.375)$ & 0.4627 \\
\hline $7: 75348306$ & rs11770686 & $\mathrm{T} / \mathrm{A}$ & HIP1 & 0.474 & 0.497 & $1.027(0.8477-1.244)$ & 0.7862 \\
\hline $8: 18308810$ & rs10109552 & $\mathrm{G} / \mathrm{T}$ & Intergenic & 0.276 & 0.258 & $1.03(0.8285-1.28)$ & 0.7916 \\
\hline $10: 66483216$ & rs1915613 & $\mathrm{C} / \mathrm{T}$ & Intergenic & 0.244 & 0.240 & $0.9068(0.7207-1.141)$ & 0.4042 \\
\hline 10:68845715 & rs12764057 & $\mathrm{T} / \mathrm{G}$ & CTNNA3 & 0.419 & 0.418 & $0.9336(0.7649-1.14)$ & 0.4996 \\
\hline $10: 68850419$ & rs10822974 & $\mathrm{A} / \mathrm{G}$ & CTNNA3 & 0.496 & 0.497 & $0.9439(0.7716-1.155)$ & 0.5748 \\
\hline $10: 68917164$ & rs7903491 & $\mathrm{G} / \mathrm{A}$ & CTNNA3 & 0.414 & 0.400 & $1.112(0.9092-1.359)$ & 0.3019 \\
\hline 11:35329615 & rs3794087 & $\mathrm{G} / \mathrm{T}$ & SLC1A2 & 0.240 & 0.254 & $0.9561(0.7662-1.193)$ & 0.6911 \\
\hline $12: 28974648$ & rs10843247 & $\mathrm{T} / \mathrm{C}$ & Intergenic & 0.286 & 0.259 & $1.094(0.8785-1.361)$ & 0.4234 \\
\hline $15: 77963887$ & rs9652490 & $\mathrm{A} / \mathrm{G}$ & LINGO1 & 0.200 & 0.195 & $0.982(0.771-1.251)$ & 0.8832 \\
\hline $15: 77972770$ & rs11856808 & $\mathrm{T} / \mathrm{C}$ & LINGO1 & 0.340 & 0.304 & $1.139(0.924-1.404)$ & 0.2225 \\
\hline 18:59274791 & rs11152303 & $\mathrm{G} / \mathrm{A}$ & Intergenic & 0.255 & 0.241 & $1.087(0.8681-1.361)$ & 0.4671 \\
\hline
\end{tabular}

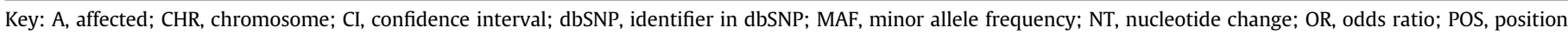
(hg19); U, unaffected. 
Although the present study does not support a common role for genetic variants in PD and ET, further study is required to understand the potential link between PD and ET.

\section{Disclosure statement}

All authors report no conflict of interests.

\section{Acknowledgements}

The authors thank the patients and controls for their participation in the study. This work was financially supported by the Michael J. Fox Foundation. GAR holds a Canada Research Chair in Genetics of the nervous system and the Wilder Penfield Chair in Neurosciences. We thank Daniel Rochefort, Pascale Hince, Helene Catoire, Cynthia Bourassa, Pierre Provencher, Cathy Mirarchi, and Vessela Zaharieva for their assistance. We thank the Quebec Parkinson's Network and its members (http://rpq-qpn.ca/) for their collaboration and Michael J. Fox Foundation for Parkinson's research.

\section{Appendix A. Supplementary data}

Supplementary data associated with this article can be found, in the online version, at https://doi.org/10.1016/j.neurobiolaging.2018. 01.001.

\section{References}

Algarni, M., Fasano, A., 2017. The overlap between essential tremor and Parkinson disease. Parkinsonism Relat. Disord. 46 Suppl 1, S101-S104.

Lees, A.J., Hardy, J., Revesz, T., 2009. Parkinson's disease. Lancet 373, 2055-2066.

Muller, S.H., Girard, S.L., Hopfner, F., Merner, N.D., Bourassa, C.V., Lorenz, D. Clark, L.N., Tittmann, L., Soto-Ortolaza, A.I., Klebe, S., Hallett, M., Schneider, S.A., Hodgkinson, C.A., Lieb, W., Wszolek, Z.K., Pendziwiat, M. Lorenzo-Betancor, O., Poewe, W., Ortega-Cubero, S., Seppi, K., Rajput, A., Hussl, A., Rajput, A.H., Berg, D., Dion, P.A., Wurster, I., Shulman, J.M. Srulijes, K., Haubenberger, D., Pastor, P., Vilarino-Guell, C., Postuma, R.B., Bernard, G., Ladwig, K.H., Dupre, N., Jankovic, J., Strauch, K., Panisset, M., Winkelmann, J., Testa, C.M., Reischl, E., Zeuner, K.E., Ross, O.A., Arzberger, T., Chouinard, S., Deuschl, G., Louis, E.D., Kuhlenbaumer, G., Rouleau, G.A., 2016 Genome-wide association study in essential tremor identifies three new loci. Brain 139 (Pt 12), 3163-3169.

Rocca, W.A., Bower, J.H., Ahlskog, J.E., Elbaz, A., Grossardt, B.R., McDonnell, S.K. Schaid, D.J., Maraganore, D.M., 2007. Increased risk of essential tremor in first-degree relatives of patients with Parkinson's disease. Mov. Disord. 22, $1607-1614$ 